Rapid and Accurate detection of Pseudomonas aeruginosa by real-time PCR with Melting Curve Analysis targeting gyrB gene

Maiko Motoshima ${ }^{a}$, Katsunori Yanagihara ${ }^{\mathrm{a} *}$, Kazuko Fukushima ${ }^{\mathrm{a}}$, Sayaka Mori ${ }^{\mathrm{a}}$, Kazuyuki Sugahara ${ }^{\mathrm{a}}$, Yohichi Hirakata ${ }^{\mathrm{a}}$, Yasuaki Yamada ${ }^{\mathrm{a}}$, Shigeru Kohno ${ }^{\mathrm{b}}$ and Shimeru Kamihira $^{\mathrm{a}}$

${ }^{a}$ Department of Laboratory Medicine, ${ }^{b}$ Second Department of Internal Medicine, Nagasaki University Graduate School of Biomedical Sciences, and Central Diagnostic Laboratory, Nagasaki University Hospital

1-7-1, Sakamoto, Nagasaki City, Nagasaki, Japan 852-8501

Address correspondence to: K. Yanagihara, Department of Laboratory Medicine, Nagasaki University Graduate School of Biomedical Sciences,

1-7-1, Sakamoto, Nagasaki City, Japan 852-8501

Tel: +81-95-849-7418, Fax: +81-95-849-7257,

E-mail:k-yanagi@nagasaki-u.ac.jp

Running title: Real-time PCR with MCA for P. aeruginosa 


\begin{abstract}
Laboratory detection of Pseudomonas spp, particularly P. aeruginosa is an important assay in the nosocomial control. The study was designed firstly to establish a new assay applied LightCycler PCR technology with melting curve analysis (MCA). A total of 224 gram-negative isolates were used to verify the assay system. The PCR with MCA method using the P. aeruginosa-specific gyrase B gene primers was rapid and accurate; the total run is approximately 3 hours and the sensitivity and specificity relative to the Vitek results were $98.1 \%$ and $100 \%$, respectively. Vitek identificaton system was not able to identify the isolates from the new P. otitidis species opposite to the Real-time PCR.
\end{abstract}

. This assay was validated to be accurate with an overall sensitivity and specificity of 98.7\% and 98.9\%. Conclusively, this rapid and accurate PCR assay with MCA will help to manage and control infections with $P$. aeruginosa.

Key words: Pseudomonas, melting temperature, taxonomy, gyrase B

\title{
Introduction
}

Strains of Pseudomonas (P.) aeruginosa are one of the most important pathogens, because they are often isolated from various clinical specimens, are resistant to anti-bacterial agents (especially multidrug-resistant), and are common as an important nosocomial pathogen with diverse phenotypes (Pollack 2000). In particular, the bacterium is noted to be the fourth most commonly-isolated nosocomial pathogen, accounting for $10.1 \%$ of all hospital-acquired infections according to the CDC. In our hospital, $9.4 \%$ of 7,152 bacterial isolates for one year of 2005 were P. aeruginosa, implying the third rank following the Staphylococcus aureus of $10.3 \%$ and the E. coli of 
$10.1 \%$. Since they are usually isolated from compromised patients carrying severe diseases, rapid and precise detection and adequate therapy for the infections are critical for successful outcomes for such patients. All of these issues stress the value of studying how to rapidly and precisely detect $P$. aeruginosa. The current strategy of bacterial identification is now biochemical testing, consisting of primary detection with various culture media and identification using commercial identification systems, such as a Vitek system (bioMerieux, Hazelwood, MO, USA). However, such procedures are not only time- and labor-consuming, but also problematic in diagnostic specificity. Therefore, to overcome these problematic issues, efforts aimed at the species-specific detection of bacterial DNA have been made. For the genetic identification and characterization of bacteria, the $16 \mathrm{~S}$ rRNA gene is most commonly used, but we pay attention to the $\operatorname{gyr} B$ gene, because it is rarely transmitted horizontally, its molecular evolution rate is higher than that of 16SrRNA, and the gene is distributed ubiquitously (Anzai et. al, 2000; Kasai et al, 2006). Thus, general bacterial classification is phylogeny based, so that phenetic-based clinical classification of the Pseudomonads has been reported to require consideration of the relationships of the various subbranches (Vandamme et al, 1996). In particular, the laboratory detection of Pseudomonas depends upon the vague and broad definition, subsequently the discrepant results have been reported between standard biochemical and new genetic tests (Qin et. al, 2003; Clarke et al, 2003). Accordingly, to improve the PCR procedures for the genetic detection of $P$. aeruginosa using the $P$. aeruginosa gyrB-specific primers, we applied a LightCycler Technology with high-speed amplification and a melting curve analysis (MCA). Interestingly, the present study happened to present a chance of discovering a novel pseudomonas of $\mathrm{P}$. otitidis being mistaken for P. aeruginosa. Accordingly, we here introduce a rapid and accurate genotypic identification procedure by PCR with MCA and discuss the laboratory and clinical relevancy of the genetic assay method. 
Materials and Methods

Clinical samples and isolates:

A total of 224 isolates of gram-negative bacteria randomly selected from our stock libraries isolated at the clinical microbiology laboratory, Nagasaki University Hospital from Jan. 2005 to May 2006, were used for the present study. All isolates were identified according to a standard biochemical identification method using standard culture media and a commercial Vitek system (bioMerieux, Hazelwood, Mo, USA) (Kis ka and Gilligan 2003). Briefly, after gram staining, specimens were processed and plated on primary standard media, such as BTB medium (KANTO Chem. Co. Inc, Tokyo, Japan), Chocolate-agar and blood-agar plates (NISSUI Pharmaceutical Co., LTD, Tokyo, Japan). Gram-negative isolated colonies were subcultured onto blood-agar/MacConkey-agar/PASA-agar triplates (Davis et al, 1983) (PASA; P. aeruginosa selective agar including 9-chloro-9-(4-diethlyaminophenyl)-10-phenylacridan. BD, Franklin Lakes, NJ, USA) and were checked for positivity of oxidation and others. Colonies were considered to be P. aeruginosa when negative for the gram stain and positive for typical morphology, oxidation, PASA, growth at $42^{\circ} \mathrm{C}$, being a lactose nonfermenter, and blue-green color (the $1^{\text {st }}$ step identified isolates). If at least one of the items described above is disagreed, final identification was decided by the test results based on the Vitek system with a gram-negative identification card (the $2^{\text {nd }}$ step identified isolates). Then, 108 clinical sputum specimens freshly obtained from patients with respiratory infections were subjected for direct identification using the new genetic test.

\section{DNA isolation:}

Bacterial DNA was extracted from bacteria solution of the 0.5 McFarland concentration using the boiling method (Sulzinski et al, 1997). The DNA extraction from practical clinical specimens was performed using the QIAamp DNA Mini Kit 
(QIAGEN, Valencia, CA, USA) according to the manufacturer's instruction. Five $\mu \mathrm{l}$ aliquots of extracted DNA solution were used as the PCR template DNA.

\section{Real-Time PCR/MCA assay:}

For amplifying the $P$. aeruginosa gyrB gene, a LightCycler thermal cycle instrument (Roche Applied Science, Mannheim, Germany) equipped with the MCA analysis programme was employed using the primers, cct gac cat ccg tcg cca caa c (gyrB-398) and cgc agc agg atg ccg acg cc (gyrB-620) corresponding to nucleotides 398-620 of the gene (accession No NC_002516) (Qin et al, 2003). Reactions were performed in a $20 \mu \mathrm{l}$ volume with $0.5 \mu \mathrm{M}$ primers, $5 \mu \mathrm{DNA}$ template, $0.25 \mu \mathrm{M}$ of the LightCycler FastStart DNA Master SYBR Green I kit (Roche Applied Science, Mannheim, Germany) plus GC Melt (Clontech Laboratories, Mountain View, USA ) and DMSO (Wako Co., Lmt.,Osaka, Japan). The reaction conditions were $95^{\circ} \mathrm{C}$ for 10 min for activation of the Taq polymerase and then 35 cycles of $10 \mathrm{sec}$ each at $96^{\circ} \mathrm{C}$ (denaturation) followed by $12 \mathrm{sec}$ at $72^{\circ} \mathrm{C}$ (annealing and extension). After 35 amplification cycles, according to the LightCycler System, the PCR product annealed at $65^{\circ} \mathrm{C}$ for 10 seconds was raised from $65^{\circ} \mathrm{C}$ to $96^{\circ} \mathrm{C}$ at a transition rate of $0.2^{\circ} \mathrm{C}$ during continuous fluorescence monitoring at $640 \mathrm{~nm}$ after excitation at $483 \mathrm{~nm}$. Fluorescence data were analyzed using the LightCycler software (version 3.5, Roche Diagnostics).

To establish a melting temperature (Tm) specific for amplicons, the MCA analysis was conducted ten times using positive and negative controls for P. aeruginosa.

\section{Direct DNA sequencing:}

To verify the discrepant results between the current biochemical and new genetic analyses, primers were designed based on 16SrRNA gene sequence of $\mathrm{P}$. aeruginosa PAO1 (NC_002516) and they were used to amplify the 16SrDNA of the questionable strains and directly sequenced with the ABI PRISM 
Terminator Cycle Sequencing Ready Kit using an Automated DNA Sequence Analyzer (Model 310, Applied Biosystems, Foster, CA, USA). ${ }^{8)}$ Primers were designed to fractionate the 8 to 1508 region into three parts and each segement was amplified with the following conditions; $94^{\circ} \mathrm{C}$ for $2 \mathrm{~min}$; 35 cycles of $94^{\circ} \mathrm{C}$ for $30 \mathrm{sec}, 58^{\circ} \mathrm{C}$ for $30 \mathrm{sec}$ and $72^{\circ} \mathrm{C}$ for $1.5 \mathrm{~min}$. The primers consisted of 1-S: aga gtt tga tca tgg ctc ag versus 1-AS: ggc tac ctt gtt acg act, 2-R: ttc gta tta ccg cgg atg ct versus 2-F; agc agc cgc ggt aat acg aa, and 3-R; taa ggt tct tcg cgt tgc tt versus 3-F; aac gca acg cga aga acc tta. The sequence homology was compared according to the DDBJ search programme (www.ddbj.nig.ac.jp/search/blast-j.html).

\section{Results}

1) Characterization of isolates

A total of 224 isolates were subcategorized according to our criteria; 104 P.aeruginosa $\left(1^{\text {st }}\right.$ step identified isolates; 81 cases and $2^{\text {nd }}$ step identified isolates; 23) and 120 non-P.aeruginosa gram-negative bacilli. The latter 120 bacilli consisted of 27 non- $P$. aeruginosa Pseudomonas genus (4 Burkholderia. cepacia, 3 Ralstonia. pickettii, 2 P. fluorescens, 13 P. putida, 1 P. stutzeri, and 3 Comamonas.testosteroni, 1 Sphingomonas. paucimobilis) 9 A. baumannii, 8 S. maltophilia, 2 Achromobacter xylosoxidans, 1 Chryseobacterterium indologenes, 17 Klebsiella spp., 8 Citrobacter spp., 32 E. coli, 4 M. morganii, 1 Proteus mirabilis, 9 Enterobacter genus, and 2 S. marcescens.

2) Test and diagnostic validity of PCR with MCA

Using 10 positive controls of the $1^{\text {st }}$ step P. aeruginosa isolates and 10 negative control of non-P. aeruginosa gram-negative bacillus, the reproducibility and specificity of our PCR-MCA assay were verified. As shown in Fig.1, the positive controls constantly allowed a sharp peak at approximately $88^{\circ} \mathrm{C}$ (Fig $\left.1 \mathrm{~A}\right)$, but no peaks in the negative controls (Fig 1B). The within-day and between-day reproducibility of the 
melting Temperature (Tm) was acceptable with a CV of $0.7 \%$ or less. The entire run time was approximately 3.5 hours, 2.5 for the pre-analytical processing of DNA, and 0.5 hours for PCR and MCA analysis.

The lower detection level for P. aeruginosa was estimated to be approximately $10^{2}$ bacilli/ml in the dilution manner.

Next, all isolates of 224 were blindly genotyped. The results are summarized in Table 1, implying that the diagnostic sensitivity and specificity relative to standard biochemical culture methods were $98.1 \%$ and $100 \%$, respectively. As shown in Table 1 , the discrepant outcomes, positive for the Vitek and negative for the genetic test, were derived from 2 isolates (no.11 and 81) out of 23 second step isolates. The 2 isolates showed atypical colony features of morphology and a non-greenish color in the BTB agar plate. The Vitek assay revealed positive data for $P$. aeruginosa with a probability of 93-93.5\% according to the Vitek scoring system. However, the system pointed out and warned the negative utilization of D-mannose and positive utilization of ELLM which the findings are unusual for $P$. aeruginosa (Funke G et. al, 2004). The phenetic characteristic features of the 2 isolates are summarized in Table 2.

To confirm which is correct, the 16SrRNA gene was sequenced in the two isolates with discrepant outcomes and one isolate (no.55) with typical findings as a positive control. As shown in Fig.2, the sequence homology for P. aeruginosa (NC_002516, Gene ID:3240211) was $100 \%$ in the positive control, $98.642 \%$ ( 20 substitution of $1473 \mathrm{bps}$ ) in the no.11 strain, and $98.713 \%$ (19 of $1476 \mathrm{bps})$ in the no. 81 strain, respectively. On the other hand, the BLAST-SEARCH (DDBJ) hit a novel Pseudomonas species of Pseudomonas otitidis (AY-953147) with nucleotide substitution of only 2 bases in the no. 11 strain and with complete accordance in the no. 81 strain.

Then, the diagnostic accuracy of the PCR assay for primary identification was validated using 108 clinical specimens. As shown in Table 3, the culture media grew 258 strains from 108 specimens consisting of 54 P.aeruginosa from 54 specimens, 89 
other causative bacilli and 65 indigenous bacteria. The PCR assay gave positive signals against $\mathrm{P}$. aeruginosa in 56 specimens of the same 54 specimens as that of the culture and additional 2 specimens. The 2 specimens grew only indigenous bacterium in one specimen and non-P.aeruginosa GNR in the other one.

By using the above mentioned isolates and clinical specimens, the PCR-MCA assay was validated based on the results with the standard culture method. Table 4 shows high accuracy, with an overall sensitivity and specificity of $98.7 \%$ and $98.9 \%$.

\section{Discussion}

Laboratory detection of $P$. aeruginosa remains an important assay in the patient management and nosocomial infection control. Nowadays, regardless of the vague phenetic definitions for a large number of Pseudomonas spp., identification of bacterial species in hospital microbiology laboratories is mainly based on morphology and biochemical approaches. Moreover, such current methodology remains to be elucidated, especially regarding rapidity, accuracy, objectivity and cost-performance in the detection technology. Recently, the concept of polyphasic taxonomy (Vandamme 1996; Vandamme 2003 which is a classification method based on the data integrating genetic, phenetic and phylogenetic information, is now being introduced into practical microbiology laboratories, implying that genetic testing is indispensable, even at hospital laboratories. Accordingly, we established a new and feasible set of real-time PCR with MCA for the detection of $P$. aeruginosa probably contributing to the resolution of the above problematic aspects. To delineate bacterial species, we used the conserved region of the gyr B gene. Currently, the 16S rRNA, groE, opr and exotoxin genes have been employed as genetic markers for PCR amplification (De Vos et al, 1997; Kurupati et al, 2005; Khan and Cerniglia, 1994). Although the PCR detection methods using such genes have been reported to be sensitive and specific, the gyrB gene coding a type II topoisomerase has recently been noted to be a better candidate for the 
identification of bacterial species than 16SrRNA of the standard gene, as described in the Introduction. Indeed, our PCR results, using the gyrB primers, offered an excellent validity for the detection and identification of only P. aeruginosa species with the $98 \%$ sensitivity and $100 \%$ specificity relative to the standard method. The reason why the sensitivity was not $100 \%$ was demonstrated to result from the false positive in the Vitek, indicating that the genetic assay is superior. The two isolates discovered, owing to the discrepant results, were confirmed to be a novel Pseudomonas species of $P$. otitidis sp. Nov (Clarke et al, 2006). (accession no AY953147) by 16SrRNA sequencing. In other words, the PCR-MCA assay has been shown to be able to discriminate P. otitidis from $P$. aeruginosa. This $P$. otitidis, recently discovered as a causative agent of optic infection, is closely related to, but different from, $P$. aeruginosa. The type strain of $P$. otitidis has been designated as $\mathrm{MCC} 10330^{\mathrm{T}}$. Our isolates of $\mathrm{P}$. otitidis were also isolated from patients with otitis, and their phenetic characteristics were similar to that of the reports $^{13)}$. All of these indicate the necessity of polyphasic examinations, even for routine bacterial identification, in particular for the genus Pseudomonas, because many Pseudomonas species are now being reclassified into separate genera (Vandamme et al, 1996).

Then, the detection sensitivity was $10^{2}$ bacilli/ $/ \mathrm{ml}$, equivalent to the previous reports (Kurupati et al, 2005; Khan and Cerniglia, 1994; Davis et al, 1983). The turn-around-time and labor were also reduced, because the entire run is approximately 3 hours. Considering such characteristics of the assay, we studied whether it is acceptable for the primary detection of P. aeruginosa in clinical specimens. Compared to the outcome obtained by the culture test, the PCR test gave a satisfactory diagnostic validity with $100 \%$ sensitivity and $96 \%$ specificity. Two specimens were discrepant, positive PCR but negative culture. This discrepancy may be related to a property of the PCR assay, in that it can detect dead P.aeruginosa or a small number of P. aeruginosa that would be under the detection limit of the media test. 
Although the genetic test itself is usually estimated to be experimentally significant, it is not practical to apply such a genetic test targeting only one bacillus for all routine specimens. Accordingly, we are now organizing the allotment of roles of culture and genetic tests. That is, the genetic assay is restrictively subjected to emergent specimens and atypical isolates corresponding to the $2^{\text {nd }}$ step of the Vitek.

Finally, this PCR-MCA assay appears promising for the accurate and rapid detection of P. aeruginosa in various clinical specimens. At present, an important point is to immediately implement the genetic test under the consideration of scientific and economical aspects.

Acknowledgements

Supported in part by grants (No.17390165/No.15659136) from the Ministry of Education, Culture, Sports, Science, and Technology of Japan

\section{References}

Anzai Y, Kim H, Park JY, Wakabayashi H, Oyaizu H (2000) Phylogenetic affiliation of the pseudomonads based on $16 \mathrm{~S}$ rRNA sequence. Int J Syst Evol Microbiol 50(4):1563-89.

Clarke L, Moore JE, Millar BC, Garske L, Xu J, Heuzenroeder MW, Crowe M, Elborn JS (2003) Development of a diagnostic PCR assay that targets a heat-shock protein gene (groES) for detection of Pseudomonas spp. In cystic fibrosis patients. J Med Microbiol 52:759-763.

Clark LL, Dajcs JJ, McLean CH, Bartell JG, Stroman DW (2006) Pseudomonas otitidis sp. Nov, isolated from patients with otic infections. Int J Syst Evol Microbiol 56:709-714.

Davis JR, Stager CE, ArajGF (1983) 4-h Identification of Pseudomonas aeruginosa with 9-Chloro-9-(4-Diethylaminophenyl)-10-Phenylacridan. J Clin Microbiol 
17(6):1054-1056.

De Vos D, Lim A J, Pirnay JP, Struelens M, Vandenvelde C, Duinslaeger L, Vanderkelen A, Cornelis P (1997). Direct detection and identification of pseudomonas aeruginosa in clinical samples such as skin biopsy specimens and expectorations by multiplex PCR based on two outer membrane lipoprotein genes, oprI and oprL. J Clin Microbiol 35(6): 1295-9.

Funke G, Funke-Kissling P (2004). Evaluation of the new VITEK 2 card for identification of clinically relevant gram-negative rods $\mathrm{J}$ Clin Microbiol. Sep;42(9):4067-71.

Kurupati P, Kumarasinghe G, Laa Poh C (2005) Direct identification of Pseudomonas aeruginosa from blood culture bottles by PCR-enzyme liked immunosorbent assay using oprI gene specific primers. Mol Cell Probes 19(6):417-21.

Khan AA, Cerninglia CE (1994). Detection of Pseudomonas aeruginosa from clinical and environmental samples by amplification of the exotoxin A gene using PCR. Appl Environ Microbiol 60(10):3739-45.

Kasai H, Watanabe K, Gasteiger E, Bairoch A, Isono K, Yamamoto S, Harayama S (1998) Construction of the gyrB database for the identification and classification of bacteria. Genome Inform Ser Workshop Genome Inform 9:13-21.

Kiska DL, Gilligan PH (2003) Pseudomonas: Manual of Clinical Microbiology, $8^{\text {th }}$ Edition, Ed Murphy PR, pp719-728, ASMpress, Washinton,D.C. USA.

Pollack M (2000) Pseudomonas aeruginosa. P1980-2003. In G.L.Mandell, J.E.Bennett, R.Dolin(ed), Principles and Practice of Infections Diseases, $5^{\text {th }}$ ed. Churchill Livingstone Inc., New Yowk, N.Y.

Qin X, Emerson J, Stapp J, Stapp L, Abe P, Burns JL (2003) Use of real-time PCR with multiple targets to identify pseudomonas aeruginosa and other nonfermenting gram-negative bacilli from patients with cystic fibrosis. J Clin Microbiol 41(9):4312-4317. 
Sulzinski MA, Moorman GW, Schlagnhaufer B, Romaine CP (1997) A simple DNA extraction method for PCR-based detection of Xanthomonas campestris pv. Pelargonii in geraniums. J Phytopathol 145:213-215.

Vandamme PAR (2003) Taxonomy and classification of Bacteria: Manual of Clinical Microbiology, $8^{\text {th }}$ Edition, Ed Murphy PR, pp271-286, ASMpress, Washinton,D.C. USA.

Vandamme P, Pot B, Gillis M, De Vos P, Kersters K, Swings J (1996) Polyphasic Taxonomy, a consensus approach to bacterial systematics. Microbiological Reviews 60(2):407-438. 
Legends

Figure 1: Melting curve analysis (MCA) of gram-negative bacilli,

(A)P.aeruginosa positive samples provided the sharp peaks with a constant Tm of $87.9{ }^{\circ} \mathrm{C}$ and (B) non-P. aeruginosa samples allowed no peak.

Figure 2: Comparison of sequence homology among P.aeruginosa PA01 and the 2 strains of No. 55 and 81.

The BLAST-SEARCH (DDBJ) hit a novel Pseudomonas species of Peusodomanas otitidis (AY-953147) with nucleotide substitution of only 2 bases in the no. 11 strain and with complete accordance in the no. 81 strain. 
(A) $P$. aeruginosa

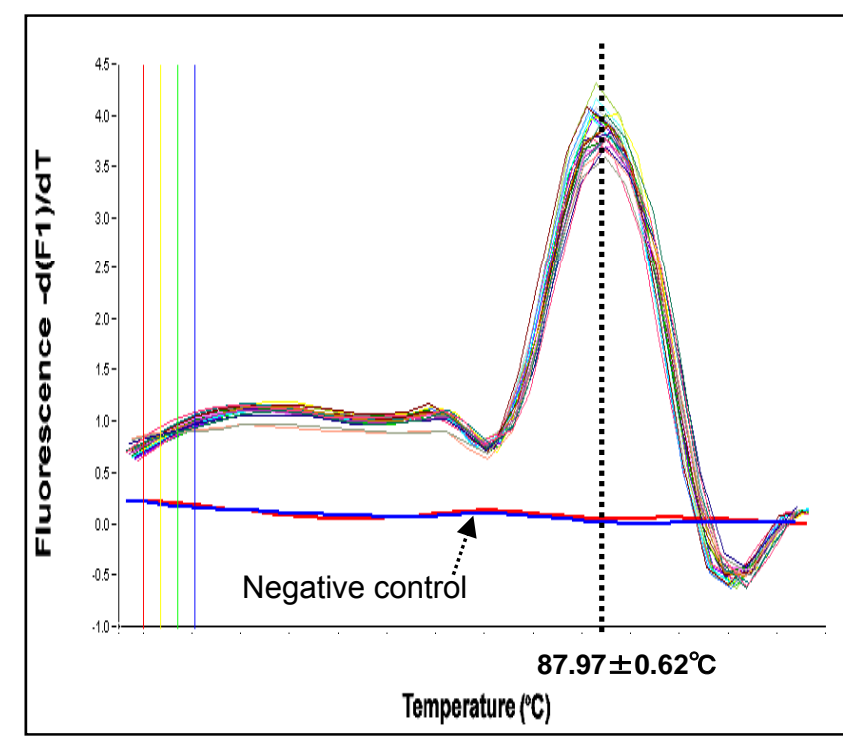

Fig. 1.Motoshima et al.
(B) gram-negative bacilli except for $P$. aeruginosa

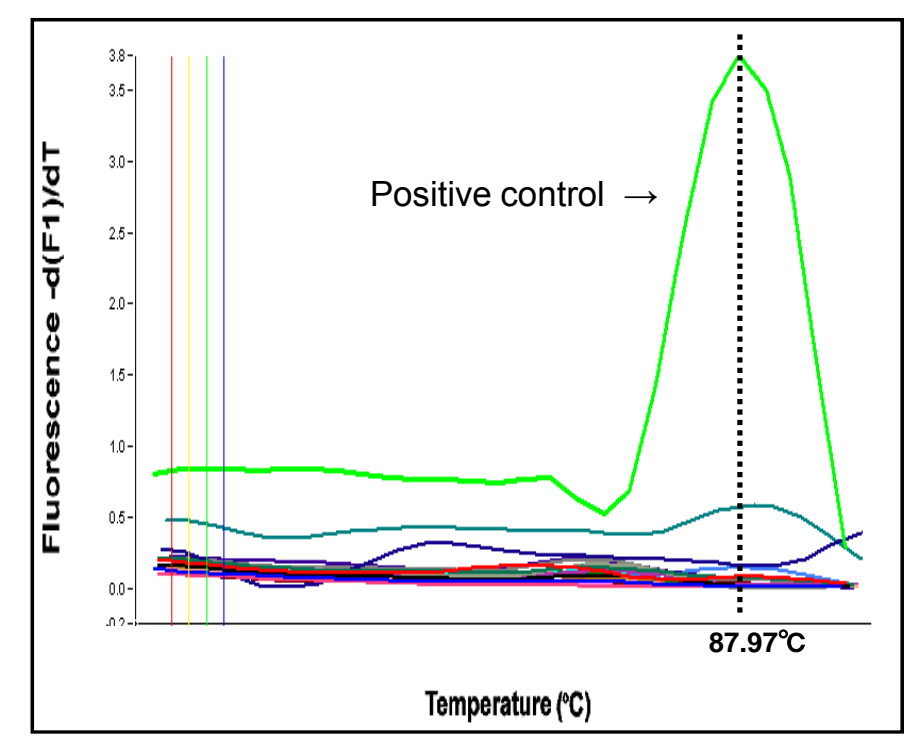


Table 1. Comparison of the outcomes between the standard biochemical culture method and PCR-MCA assay for 224 isolates of gram-negative rods.

\begin{tabular}{|c|c|c|c|c|}
\hline \multirow{2}{*}{$\begin{array}{l}\text { Bacterial strain } \\
\text { Pseudomonas aeruginosa }\end{array}$} & & \multirow{2}{*}{$\begin{array}{c}\begin{array}{l}\text { Number } \\
\text { of strains }\end{array} \\
104\end{array}$} & \multicolumn{2}{|c|}{ gyrB-PCR (\%) } \\
\hline & & & 102 & $(98.1 \%)$ \\
\hline 1st step identification & & 81 & 81 & $(100 \%)$ \\
\hline 2nd step identification & & 23 & 21 & $(91.3 \%)$ \\
\hline VITEK2 identification \% & $\geqq 99 \%$ & 48 & 48 & $(100 \%)$ \\
\hline & $<99 \% \geqq 96 \%$ & 48 & 48 & $(100 \%)$ \\
\hline & $<96 \% \geqq 93 \%$ & 8 & 6 & $(75 \%)$ \\
\hline Genus Pseudomonas & & 27 & 0 & $(0 \%)$ \\
\hline Burkholderia cepacia & & 4 & 0 & $(0 \%)$ \\
\hline Ralstonia pickettii & & 3 & 0 & $(0 \%)$ \\
\hline Pseudomonas fluorescens & & 2 & 0 & $(0 \%)$ \\
\hline Pseudomonas putida & & 13 & 0 & $(0 \%)$ \\
\hline Pseudomonas stutzeri & & 1 & 0 & $(0 \%)$ \\
\hline Comamonas testosteroni & & 3 & 0 & $(0 \%)$ \\
\hline Sphingomonas paucimobili & & 1 & 0 & $(0 \%)$ \\
\hline Other gram-negative bacteria & & 93 & 0 & $(0 \%)$ \\
\hline total & & 224 & & \\
\hline
\end{tabular}




\section{Pseudomonas aeruginosa PA01 ACCESSION NC_002516 GeneID: 3240211}

No. 11

No. 81

1 gaactgaaga gtttgatcat ggctcagatt $\ldots \ldots \ldots \ldots \ldots \ldots \ldots \ldots \ldots \ldots \ldots \ldots \ldots \ldots \ldots \ldots$

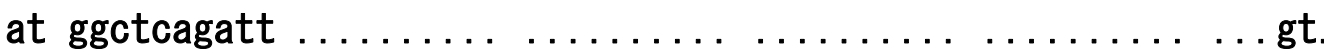

ggctcagatt $\ldots \ldots \ldots \ldots \ldots \ldots \ldots \ldots \ldots \ldots \ldots \ldots \ldots \ldots \ldots \ldots$

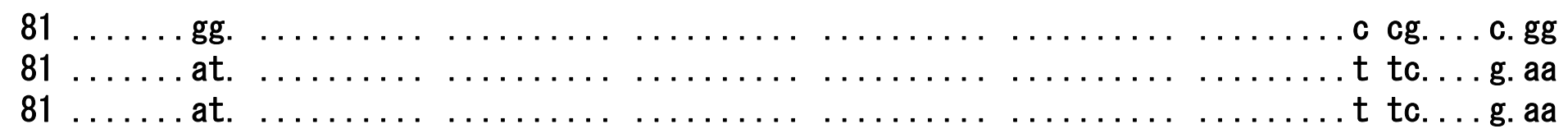

$\begin{array}{llll}161 & \ldots \ldots \ldots \ldots & \ldots \ldots \ldots & \text { ga. } \\ 161 & \ldots \ldots \ldots & \ldots \ldots \ldots & \text { ac. }\end{array}$

$161 \ldots \ldots \ldots \ldots+\cdots \cdots \cdots+$ ac

$241 \ldots \ldots \ldots \ldots \ldots$ a. . . t.

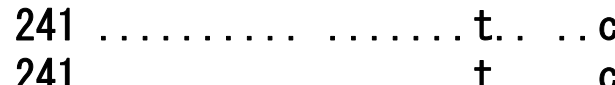

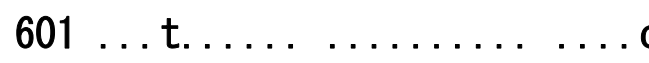

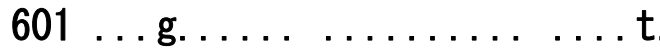

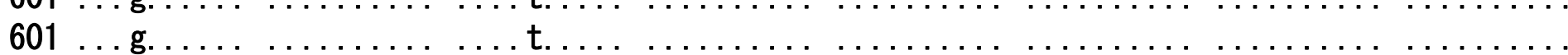

$961 \ldots \ldots \ldots \ldots$ t. $\ldots$ t. $\ldots \ldots \ldots \ldots \ldots \ldots \ldots \ldots \ldots \ldots \ldots \ldots \ldots \ldots$

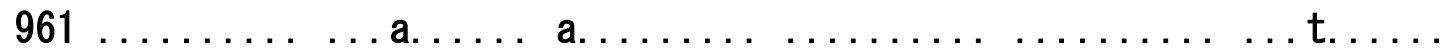

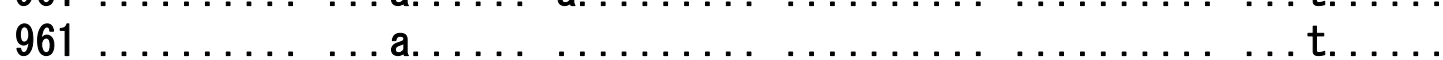

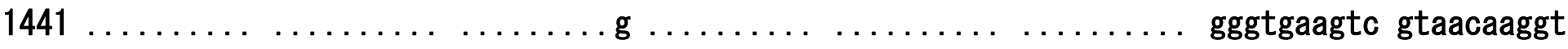

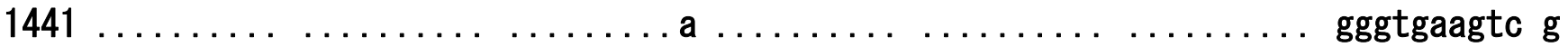

$1441 \ldots \ldots \ldots \ldots \ldots \ldots \ldots \ldots \ldots \ldots \ldots \ldots \ldots \ldots \ldots \ldots$ gggtgaagtc gtaaca

Fig. 2 Motoshima et al. 
Table 2. Characterization of the two strains of $P$. otitidis isolated in the present study

\begin{tabular}{|c|c|c|c|}
\hline \multirow[b]{2}{*}{ items } & \multirow[t]{2}{*}{ P. aeruginosa } & \multicolumn{2}{|c|}{ P. otitidis } \\
\hline & & no. 11 & no.81 \\
\hline $\begin{array}{l}\text { Gram(-) rod } \\
\text { Growth }\end{array}$ & yes & yes & yes \\
\hline on MacConkey & yes & yes & yes \\
\hline on PASA & yes & no & no \\
\hline at $42^{\circ} \mathrm{C}$ & yes & yes & yes \\
\hline Oxidation(oxidase) & yes & yes & yes \\
\hline Pyocyanin & yes & no & no \\
\hline \multicolumn{4}{|l|}{ Utilization of } \\
\hline$\alpha-D-L a c t o s e$ & yes & no & no \\
\hline D-mannose & yes & no & no \\
\hline ELLM* & no & yes & yes \\
\hline
\end{tabular}

${ }^{*}$;nitrobenzoic acid 
Table 3: Comparison of isolation profiles by the culture and PCR assays.

The culture media grew 258 strains from 108 specimens consisting of 54 P.aeruginosa, 89 others, and 65 Indigenous bacteria, while the PCR reacted the same 54 specimens as that of the culture and additional 2 specimens.
main isolated bacteria from 108 clinical samples
culture
PCR-MCA

Pseudomonas aeruginosa

54

54

Pseudomonas fluorescens

Burkholderia cepacia

$1 \quad 0$

Other gram-negative bacteria.

Haemophilus influenzae

1

33

0

Moraxella catarrhalis

3

2

0

0

Staphylococcus aureus

$\begin{array}{cc}23 & 0 \\ 6 & 0 \\ 1 & 0 \\ 2 & 0\end{array}$

Genus Candida

17

0

indigenous bacteria*)

65

1

*)Nonpathogenic Streptococcus, Neisseria, Haemophilus, CNS(coagulase-negative staphylococcus )and GPR(Gram positive rod) were included in resident microbiota. 
Table 4: Summary of a diagnostic validity of the PCR-MCA relative to the results with the standard culture method by using 224 isolates and 108 clinical specimens

\begin{tabular}{lcccccc}
\hline subjects & No & \multicolumn{2}{c}{ positive for P.aeruginosa } & & \\
\cline { 3 - 4 } & & culture & PCR & & sensitivity & specificity \\
\hline Isolates & 224 & 104 & 102 & $98.1 \%$ & $100 \%$ \\
Specimens & 108 & 54 & 56 & $100 \%$ & $96.4 \%$ \\
\cline { 5 - 6 } Overall & 332 & 158 & 158 & $98.7 \%$ & $98.9 \%$ \\
\hline
\end{tabular}

